\title{
Detecting Spatially Consistent Structural Differences in Alzheimer's and Fronto Temporal Dementia Using Deformation Morphometry
}

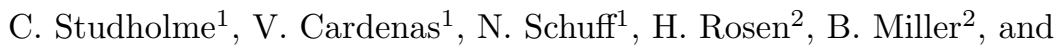 \\ M. Weiner ${ }^{1}$
}

1 Dept. Radiology, U.C.S.F., VAMC (116S), 4150 Clement Street, San Francisco.

2 Memory and Aging Center,Dept. Neurology, U.C.S.F., San Francisco

cs10itsa.ucsf.edu

\begin{abstract}
Atrophy is known to occur at specific sites around the brain in both Alzheimer's disease (AD) and Fronto-Temporal Lobe Dementia (FTLD), inducing characteristic shape changes in brain anatomy. In this paper we employ an entropy driven fine lattice free form registration algorithm to investigate whole brain structural changes induced by these diseases relative to normal anatomy, using deformation morphometry. We focus on Alzheimer's disease (AD) and two common sub groups of FTLD: the frontal lobe variant (FTD) and semantic dementia (SD). The shape of each subject group was characterized at each point in the reference anatomy by the distribution of the determinant of the Jacobian of the transformation mapping the subject to a common reference anatomy. Statistical measures were then applied to locate points where voxel level differences in the Jacobian occur between a control group and each of the disease groups, indicating spatially consistent shape differences induced by a particular disease. Spatial maps of the statistical differences showed very different structural characteristics in each disease. AD was characterized by relative contractions in regions of the hippocampus and the parietal lobe and expansions of the ventricular CSF spaces. FTD was characterized by patterns of contraction in the frontal lobe. SD was characterized by large contractions in the temporal lobe, hippocampus and expansion of the ventricular CSF spaces.
\end{abstract}

\section{Introduction}

Alzheimer's disease (AD) and fronto-temporal lobe dementia (FTLD) are two forms of dementia with distinctly different etiologies but with clinical symptoms that are often difficult to distinguish. Therefore, improvements in the differential diagnosis between AD and FTLD are desirable for better staging of patients in drug trials and treatment planning. Computational shape analysis of structural MRI data that identifies anatomical changes in the brain has the potential to aid a differential diagnosis. Partially driven by the development of more accurate anatomical registration techniques, the field has expanded rapidly over 
the last few years. Much of the early work concentrated on specific anatomical regions such as the corpus callosum [7] which has remained an active area of methodological development 20. Region based analysis techniques can rely on the identification and mapping of points on a specific anatomical boundary for statistical shape analysis [4]14 and has been used to analyze regional shape variation such as in the hippocampus in AD 6. Whole brain approaches have concentrated on the analysis of the volume transformation describing the non rigid mapping between subjects and a reference anatomy [127]. A recent study of schizophrenia [10] analyzed low resolution 3D deformation fields and detected effects of disease without a-priori assumptions. Related techniques, such as voxel morphometry [2], which examine local changes in the regional density of particular tissue types, have detected significantly different structural patterns in degenerative diseases 22 .

In this work we focus on purely deformation based morphometry relying on accurate registration to provide spatial transformations describing the subtle differences in brain shape. Specifically, we have used a high dimensional (many parameter) non-rigid free-form registration technique to map subject brains to a common reference space and then analysed the Jacobian of this transformation to look for characteristic differences in shape. We have used a voxel level Jacobian analysis to search for only those points of change which are both spatially and diagnostically consistent across the different groups.

\section{Method}

\subsection{Inter-subject Registration}

Quantification and comparison of shape differences in brain anatomy is dependent on the ability to find corresponding anatomical points in images of two different human brains. This is a fundamental problem stemming from the inherent variability of human brain anatomy, particularly in the cortex [3]. The field of non-rigid inter-subject brain mapping or atlas matching is extensive and beyond the scope of this paper. We have chosen to use a voxel based algorithm which provides fully automated registration estimates on a large range of data, primarily because the number of data-sets being examined precludes the use of perhaps more accurate methodology requiring some form of manual tracing or segmentation. Specifically we have made use of a voxel level free form deformation algorithm driven by normalised [24] mutual information [28|18] between the two MRI scans,

$$
\mathcal{C}\left(M_{1} ; M_{2}\right)=\frac{H\left(M_{1}\right)+H\left(M_{2}\right)}{H\left(M_{1}, M_{2}\right)},
$$

where $H\left(M_{1}\right)$ and $H\left(M_{2}\right)$ are the marginal entropies of the reference $m_{1} \in M_{1}$ and subject $m_{2} \in M_{2}$ MRI values occurring in the volume of overlap of points in the reference and test subject MRI volumes, related by transformation $\mathbf{T}_{12}$. $H\left(M_{1}, M_{2}\right)$ is the joint entropy of the co-occurrence of the values in the two scans within this overlap. This provides spatial transformation estimates robust 
to tissue intensity changes which may arrise in degenerative diseases. This is a refinement of earlier registration methodology employed for tissue deformation [21] estimation and relative geometric imaging distortion 25] correction. For this application we have developed the algorithm and its implementation to provide a finer $(2.4 \mathrm{~mm})$ B-Spline lattice estimate of the mapping between different brain anatomy. Further details of the registration algorithm used here are given in 23].

\subsection{Shape Comparison}

Choice of Reference Atlas: There are a number of approaches to constructing a common reference anatomy as a target for spatial normalisation. Because we are primarily concerned with accurate normalisation of different subject groups, rather than in the ability to report results in a standard anatomical coordinate system, we have chosen to use a single subject reference MRI as opposed to an averaged and blurred MRI, thus retaining the finest anatomical structures for registration. In earlier work [23] we examined the use of our non-rigid registration methodology in mapping different subject groups to old and young anatomical atlases. This demonstrated that the consistency of spatial normalization of older, younger or atrophied brains improved when the reference atlas was roughly agematched. To age match our AD and FTLD subjects, we have chosen an older control subject (in this case a 72 year old female) as the reference space for the measurements.

Clinical Hypothesis for Shape Differences in AD and FTLD: Alzheimer's disease accelerates the loss of large projection neurons in the hippocampus [26] which may precede and is more severe than neuron loss in association cortex. MRI studies of AD [1613] show generalized brain tissue loss, reduction in GM (particularly in the parietal lobes), ventricular enlargement, and changes in T2 signal intensity. Focal atrophy of the hippocampus occurs in normal aging [11], and to a greater extent in $\mathrm{AD}$ [12. The rate of lateral ventricle enlargement (measured longitudinally), highly correlates with impaired cognition but shows variable overlap with controls [5].

In general, FTLD patients exhibit greater atrophy of anterior brain regions than $\mathrm{AD}$ and healthy elderly, while regions of the medial temporal lobe appears to be spared in FTLD [9]. FTLD, like AD, shows hippocampal atrophy [8], suggesting that sole hippocampal volume measurements may not be helpful to differentiate between FTLD and AD. Kaufer et al. 15] found a smaller anterior corpus callosum and enlarged pericallosal CSF spaces in FTLD when compared with AD. MRI also demonstrated cases with predominant temporal lobe atrophy and predominant frontal lobe atrophy in FTLD [19] supporting the existence of the temporal and frontal variants of FTLD.

Based on these previous findings, we hypothesized that our shape analysis between FTLD and AD would reveal 1) greater frontal lobe atrophy in FTLD, 2) greater anterior temporal lobe atrophy in FTLD, 3) atrophy of anterior corpus callosum and hypertrophy of pericallosal CSF spaces in FTLD, and 4) parietal lobe gray matter atrophy in AD. 
Comparing Shape in Each Group: Following registration estimation, we have a B-Spline model of the transformation $\left(x_{S}, y_{S}, z_{S}\right)=T_{R S}\left(x_{R}, y_{R}, z_{R}\right)$ mapping between the reference anatomy and each subject. This global transformation consists of a rigid component describing the patient positioning, and the remaining non-rigid components describing the global and local shape differences between each subject and the reference anatomy. To examine the shape differences only we take the approach of Davatzikos [7] and Machado [17] and examine the differential properties of the spatially normalising transformation through the Jacobian matrix,

$$
\mathbf{J}_{R S}\left(x_{R}, y_{R}, z_{R}\right)=\left[\begin{array}{lll}
\frac{\partial x_{S}}{\partial x_{R}} & \frac{\partial x_{S}}{\partial y_{R}} & \frac{\partial x_{S}}{\partial z_{R}} \\
\frac{\partial y_{S}}{\partial x_{R}} & \frac{\partial y_{S}}{\partial y_{R}} & \frac{\partial y_{S}}{\partial z_{R}} \\
\frac{\partial z_{S}}{\partial x_{R}} & \frac{\partial z_{S}}{\partial y_{R}} & \frac{\partial z_{S}}{\partial z_{R}}
\end{array}\right]
$$

of the volume transformation, between reference and subject coordinates. This matrix describes the point-wise volume change in each axis when mapping points from the reference anatomy to each subject. Large values of components indicate expansions of anatomy, while small values indicate contractions. In our methodology, the partial derivatives making up the Jacobian are evaluated analytically from the B-Spline deformation model. For the work presented in this paper we have only considered the determinant of this Jacobian $J_{R S}\left(x_{R}, y_{R}, z_{R}\right)=\left|\mathbf{J}_{R S}\left(x_{R}, y_{R}, z_{R}\right)\right|$, which is a scalar summarizing the pointwise volume change. To analyze shape statistically we first consider a single group of subject MRIs mapped to a reference MRI. At each reference coordinate $\mathbf{x}_{R}$ there will be a set of Jacobian values,

$$
\mathcal{J}_{R G}\left(\mathbf{x}_{R}\right)=\left\{J_{R 1}\left(\mathbf{x}_{R}\right), J_{R 2}\left(\mathbf{x}_{R}\right) \ldots J_{R N}\left(\mathbf{x}_{R}\right)\right\},
$$

where $N$ is the number of subjects in the group. For a given location, this set of values will provide an estimate of the distribution in the point-wise volume at that point across the group of subjects, relative to the chosen reference anatomy. For points within the gray matter of the cortex for example, this volume measure will be primarily determined by the thickness of the cortex, relative to the thickness of the cortex at that point in the reference anatomy. Globally the average Jacobian over the brain volume for each subject will describe the relative size of each subject brain relative to the target anatomy. To remove the influence of brain size from the analysis we have chosen to scale each Jacobian value by the average Jacobian of the subject. To detect differences in shape of the distributions for $\mathrm{AD} \mathcal{J}_{R A}(\mathbf{x})$, SD $\mathcal{J}_{R S}(\mathbf{x})$ and FTD $\mathcal{J}_{R F}(\mathbf{x})$ from the control group $\mathcal{J}_{R C}(\mathbf{x})$ at each point, we have used the effect size as employed by Davatzikos et al. [7] and Machado et al. [17, defined between the AD and control group as:

$$
\mathcal{E}_{C A}\left(\mathbf{x}_{R}\right)=\frac{\mu_{R A}\left(\mathbf{x}_{R}\right)-\mu_{R C}\left(\mathbf{x}_{R}\right)}{\sigma_{C \cup A}\left(\mathbf{x}_{R}\right)},
$$

where $\mu_{R A}\left(\mathbf{x}_{R}\right)$ is the mean Jacobian determinant in the Alzheimer's group and $\mu_{R C}\left(\mathbf{x}_{R}\right)$ is the mean Jacobian determinant in the control group at location $\mathbf{x}_{R}$ in the reference anatomy. The standard deviation $\sigma_{C \cup A}\left(\mathbf{x}_{R}\right)$ is derived from the combined set of Jacobian values in the control and AD group. 
Subject Imaging Data: For this study 40 Controls (17 Female, 23 Male), 31 AD (13 Female, 18 Male), 12 SD (2 Female, 10 Male), 14 FTD (3 Female, 11 Male) were imaged. All images were acquired using the same 3D gradient echo $\mathrm{T} 1$ weighted anatomical MPRAGE sequence $(\mathrm{TR}=10 \mathrm{~ms}, \mathrm{TE}=4 \mathrm{~ms})$. The images were reconstructed with a coronal slice plane at a voxel size of $1 \mathrm{~mm} \times 1 \mathrm{~mm} \times$ $1.5 \mathrm{~mm}$. Overall, dementia severity as measured by a standard minimental state examination (MMSE) test was similar in the patient groups (Control $=29 \pm 0.6$ ), $\mathrm{AD}=22 \pm 5, \mathrm{AD}=24 \pm 3, \mathrm{FTD}=24 \pm 6$; with 30 representing cognitive normal and 0 severe dementia conditions).

\section{Results}

Figure 1 shows the spatial distribution of the effect size of the Jacobian of each disease group relative to the control group overlaid onto the average control MRI, where cyan-blue indicates expansion and yellow-red is contraction of regions control subjects to disease subjects. In AD, the most prominent differences with respect to controls were contractions in the hippocampus and temporal and parietal lobe without large changes in frontal lobe. In addition, AD patients had substantially increased temporal and lateral ventricular spaces compared to controls. In FTD, the most prominent contractions when compared to controls appeared in the anterior aspects of the putamen and frontal lobe regions, while hippocampus and temporal and parietal lobes were spared. In SD, the most prominent differences from controls were bilateral expansions of the anterior temporal horn of the ventricles, accompanied by contractions in the adjacent temporal lobe regions, including hippocampus and amygdala.

\section{Discussion}

In this work we have implemented a fully automated whole brain shape analysis technique based on an entropy driven fine-lattice volume registration algorithm and applied it to identify characteristic abnormal brain structure associated with three common forms of dementia: AD, FTD and SD. Cognitive and neurobehavioral studies have indicated differences between FTLD and normal controls, and between FTLD and AD. In addition, a handful of cognitive studies have begun to identify important differences between the sub-types of FTLD, such as early impairments of executive functions in FTD and profound semantic memory breakdown in SD. Our results further support this work indicating that differences between these dementia syndromes are associated with characteristic structural changes. One important observation was that consistent spatial shape differences were detected between the patient groups that had very similar levels of dementia severity as measured by MMSE. This implies that the spatial pattern of structural abnormality reflects primarily the regional distribution of pathology as opposed to being simply associated with dementia severity. Therefore, the different spatial patterns of shape deformation could aid a differential diagnosis between these different types of dementia. 

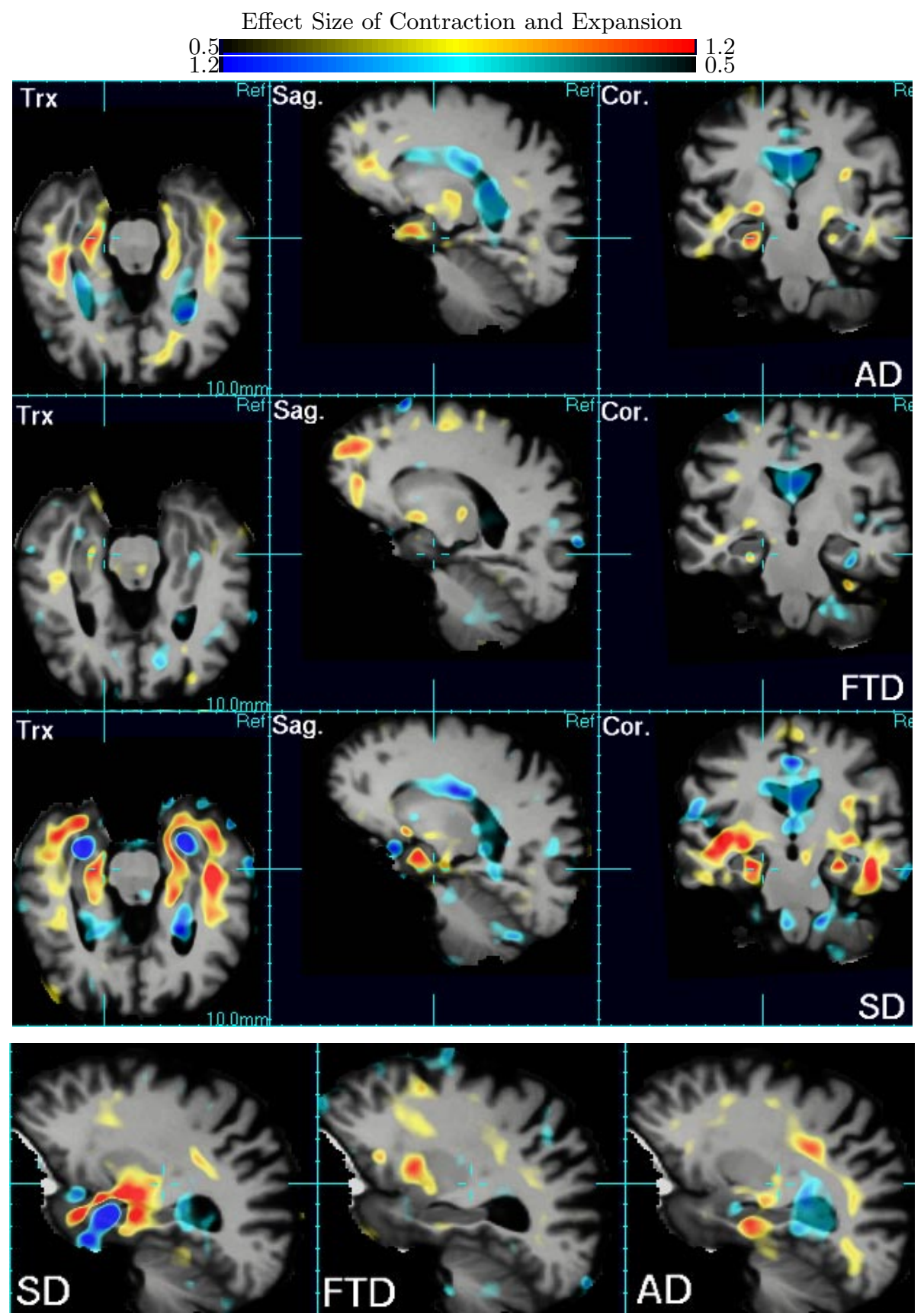

Fig. 1. Transaxial, sagittal and coronal slices through the averaged spatially normalised control group MRI $(\mathrm{N}=40)$ with colour overlay of the thresholded effect size of relative contraction (yellow-red) or expansion (cyan-blue) of tissue from the normal control group to the three disease groups. Top row shows AD, second from top FTD, third from top SD and the lower row shows a second set of sagittal slices through another plane illustrating further differences in the frontal and parietal lobe shape. 
Overall, the groups of AD and SD subjects were older than the group of FTD subjects, and age-related atrophy is well established. FTLD subjects are generally younger than $\mathrm{AD}$ subjects, making age-matching difficult. A map of structural changes due to normal aging would assist in ruling out confounding effects of age. There are many other routes to extend and improve our initial analysis, looking, for example, at the individual components, rather than simply the determinant of the Jacobian, which could provide directional information about shape differences. Methods of statistically combining shape measurements from different locations will help to improve the statistical power of this analysis 20]. Additionally, other non-parametric statistical measures may well assist in comparing shape characteristics arising from unknown sub-groups within the clinically defined diagnoses.

Acknowledgments This work was funded by a NIH grants P01 AG12435, PO1 AA11493 and R01 AG10897. The authors wish to thank D. Truran for helping select and prepare the atlas data, Dr B. Jagust for access to imaging data, Dr L. Rogers for advice on the statistical analysis and Dr J. Gee and Dr D. Pettey for discussions on the Jacobian shape analysis.

\section{References}

1. J. Ashburner and K.J. Friston. Nonlinear spatial normalization using basis functions. Neuroimage, 7(4):254-266, 1997.

2. J. Ashburner and K.J. Friston. Voxel-based morphometry-The methods. Neuroimage, 11:805-821, 2000.

3. A.J. Bartley, D.W. Jones, and D.R. Weinberge. Genetic variability of human brain size and cortical gyral patterns. Brain, pages 257-269, 1997.

4. F.L. Bookstein. Landmark methods for forms without landmarks: Morphometrics of group differences in outline shape. Medical Image Analysis, 1(3):225-243, 1997.

5. C. De Carli, J.V. Haby, J.A. Gilette D. Teichberg, S. Rapoport, and M.B. Schapiro. Longitudinal changes in lateral ventricular volume in patients with dementia of the alzheimer type. Neurology, 42:2029-2036, 1992.

6. J.G. Csernansky, L. Wang, S. Joshi J.P. Miller M. Gado D. Kido D. McKeel, J.C. Morris, and MI. Miller. Early DAT is distinguished from aging by high-dimensional mapping of the hippocampus. Neurology, 55(11):1636-1643, 2000.

7. C. Davatzikos, M. Vaillant, S.M. Resnick, J.L. Prince, S. Letovsky, and R.N. Bryan. A computerised approach for morphological analysis of the corpus callosum. Journal of Computer Assisted Tomography, 20(1):88-97, 1996.

8. R. Duara, W. Barker, and C.A. Luis. Frontotemporal dementia and alzheimer's disease: differential diagnosis. Dement Geriatr Cogn Disord, 10(1):37-42, 1999.

9. G.B. Frisoni, A. Beltramello, C. Geroldi, C. Weiss, A. Bianchetti, and M. Trabucchi. Brain atrophy in frontotemporal dementia. J Neurol Neurosurg Psychiatry, 61:157-165, 1996.

10. C. Gaser, H.P. Voltz, S. Kiebel, S. Riehemann, and H. Sauer. Detecting structural changes in whole brain based on nonlinear deformations- application to schizophrenia research. Neuroimage, 10:107-113, 1999.

11. J. Golomb, A. Kluger, and M.J. de Leon. Hippocampal formation size in normal human aging: a correlate of delayed secondary memory performance. Learning and Memory, 1:45-54, 1994. 
12. C.R. Jack, C.R. Petersen, P.C. O'Brien, and E.G. Tangalos. MR-based hippocampal volumetry in the diagnosis of alzheimer's disease. Neurology, 42:183-188, 1992.

13. T.L. Jernigan, D.P. Salmonand N. Butters, and J. Hesselink. Cerebral structure on MRI, part II: specific changes in Alzheimer's and Huntington's diseases. Biol Psychaitry, 29:68-81, 1991.

14. S. Joshi. Large Deformation Diffeomorphisms and Gaussian Random Fields for Statistical Characterization of Brain Sub-Manifolds. PhD thesis, Washington Univ., 1997.

15. D.I. Kaufer, B.L. Miller, L. Itti, L.A. Fairbanks, J. Li, J. Fishman, J. Kushi, and J.L. Cummings. Midline cerebral morphometry distinguishes frontotemporal dementia and alzheimer's disease. Neurology, 48:978-985, 1997.

16. J.S. Luxenberg, J.V. Haxby, H. Creasey, M. Sundaram, and S.I. Rapoport. Rate of ventricular enlargement in dementia of the alzheimer type correlates with rate of neuropsychological deterioration. Neurology, 37:1135-1140, 1987.

17. A.M.C. Machado, J.C. Gee, and M.F.M. Campos. Atlas warping for brain morphometry. In Proceedings of Medical Imaging 1998, pages 642-651. SPIE Press, 1998. San Diego, California.

18. F. Maes, A. Collignon, D. Vandermeulen, G. Marchal, and P. Suetens. Multimodality image registration by maximisation of mutual information. IEEE Transactions on Medical Images, 16(2):187-198, 1997.

19. R.J. Perry and J.R. Hodges. Differentiating frontal and temporal variant frontotemporal dementia from alzheimer's disease. Neurology, 54:2277-2284, 2000.

20. D.J. Pettey and J.C. Gee. Using a linear diagnostic function and non-rigid registration to search for morphological differences between poluations: An example involving the male and female corpus callosum. In Proceedings SPIE Medical Imaging, volume 4322, page In Press, 2001.

21. D. Ruckert, C. Hayes, C. Studholme, M. Leach, and D.J. Hawkes. Non-rigid registration of breast MR images using mutual information. In W. M. Wells, A. Colchester, and S. Delp, editors, Proceedings of MICCAI, pages 1144-1152, 1998. Cambridge, MA, USA.

22. A.R.B. Serge, B Rombouts, F. Barkhof, M.P. Witter, and P. Scheltens. Unbiased whole-brain analysis of gray matter loss in alzheimer's disease. Neuroscience Letters, 285:231-233, 2000.

23. C. Studholme, V. Cardenas, and M. Weiner. Multi scale image and multi scale deformation of brain anatomy for building average brain atlases. In Proceedings SPIE Medical Imaging, volume 4322, page In Press, 2001.

24. C. Studholme, D.L.G. Hill, and D.J. Hawkes. An overlap invariant entropy measure of 3D medical image alignment. Pattern Recognition, 32(1):71-86, 1999.

25. C. Studholme, E. Novotny, R. Stokking, J.S. Duncan, I.G. Zubal, and D. Spencer. Alignment of functional data acquired before and after intra-cranial electrode implantation using non-rigid MRI registration. In Proceedings of ISMRM 2000, Denver, Col., USA., page 585, 2000.

26. R.D. Terry, A. Peck, R. DeTeresa, R. Schechter, and D. Horoupian. Some morphometric aspects of the brain in senile dementia of the alzheimer type. Annals of Neurology, 10:184-192, 1981.

27. P. Thompson, R. Woods, M. Mega, and A. Toga. Mathematical/Computational challenges in creating deformable and probabilistic atlases of the human brain. Human Brain Mapping, 9:81-92, 2000.

28. W.M. Wells, P. Viola, H. Atsumi, S. Nakajima, and R. Kikinis. Multi-modal volume registration by maximisation of mutual information. Medical Image Analysis, 1(1):35-51, 1996. 